\title{
Accelerated in vitro fibril formation by a mutant $\alpha$-synuclein linked to early-onset Parkinson disease
}

\author{
Kelly A. Conway, James D. Harper \& Peter T. Lansbury \\ Center for Neurologic Diseases, Brigham and W omen's Hospital and Department of N eurology, \\ Harvard Medical School, Harvard Institutes of M edicine, 77 Ave Louis Pasteur, Boston, Massachusetts 02115, USA \\ Correspondence should be addressed to P.L.; email: Iansbury@cnd.bwh.harvard.edu
}

Two mutations in the gene encoding $\alpha$-synuclein have been linked to early-onset Parkinson's disease ${ }^{1-3}$ (PD). $\alpha$-Synuclein is a component of Lewy bodies, the fibrous cytoplasmic inclusions characteristic of nigral dopaminergic neurons in the PD brain ${ }^{4}$. This connection between genetics and pathology suggests that the $\alpha$-synuclein mutations may promote PD pathogenesis by accelerating Lewy body formation. To test this, we studied $\alpha$-synuclein folding and aggregation in vitro, in the absence of other Lewy body-associated molecules. We demonstrate here that both mutant forms of $\alpha$-synuclein (A53T and A30P) are, like wild-type $\alpha$-synuclein ${ }^{5}$ (WT), disordered in dilute solution. However, at higher concentrations, Lewy body-like fibrils and discrete spherical assemblies are formed; most rapidly by A53T. Thus, mutation-induced acceleration of $\alpha$-synuclein fibril formation may contribute to the early onset of familial PD.

In Alzheimer disease (AD), the amyloid $\beta$-protein $(A \beta)$, a chief component of the characteristic neuritic amyloid plaques, forms native-like fibrils in vitro, in the absence of other proteins associated with the disease ${ }^{6}$. The $A \beta 42$ variant fibrillizes much more rapidly in vitro than does the $A \beta 40$ variant, seeding subsequent $A \beta$ fibril growth 7 . This finding indicated a mechanistic scenario for AD pathogenesis involving accelerated fibrillization ${ }^{8}$. This proposal has gained credence with the discovery that all earlyonset familial AD (FAD) mutations result in increased $A \beta 42$ production and deposition ${ }^{9}$. The in vitro $A \beta$ fibrillization assay has also made it possible to screen for amyloid inhibitors, therapeutic candidates that will provide the ultimate test of the role of amyloid formation in $A D$ etiology. We sought to investigate a possible analogy ${ }^{10}$ between FAD and early-onset PD, by determining whether disease-associated mutations in the $\alpha$-synuclein gene product promote its fibrillization.

Bacterially-expressed recombinant WT $\alpha$-synuclein and the two mutant proteins A30P (ref. 1) and A53T (ref. 2) had indistin- guishable ‘natively unfolded’ structures (Fig. 1a, circular dichroism spectra; ref. 5). Interactions between WT and mutant proteins that may occur in vivo (all of the familial early-onset PD patients are heterozygotic for the $\alpha$-synuclein mutations ${ }^{1,2}$ ) could not be detected; 1:1 mixtures of WT with either of the mutant proteins (WT and A30P or WT and A53T) were indistinguishable from the pure protein solutions. Helical conformers could bestabilized to a similar degree in dilute solutions of all three pure proteins and the 1:1 mixtures by adding SDS (Fig. 1b), consistent with the proposal that $\alpha$-synuclein binds to lipid surfaces through amphiphilic helix formation ${ }^{11}$. At a slightly higher protein concentration and temperature $(100 \mu \mathrm{M}$ in $10 \mathrm{mM}$ $\mathrm{Na}_{2} \mathrm{HPO}_{4}, \mathrm{pH} 7.4,100 \mathrm{mM} \mathrm{NaCl}, 37^{\circ} \mathrm{C}$ ), the A53T solution showed evidence of structure formation (negative ellipticity decreased at $205 \mathrm{~nm}$ and increased at $220 \mathrm{~nm}$, data not shown) within one day, whereas neither the A30P solution nor the WT solution changed over 1 week. At even higher protein concentra-

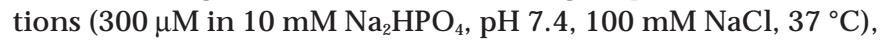
the A53T solution formed a viscous gel within one day, whereas the WT, A30P, WT/A30P, and WT/A53T solutions seemed to be unchanged over 3 weeks. Aliquots from each incubation were adsorbed onto mica and analyzed by atomic force microscopy ${ }^{12}$ (Fig. 2). The A53T incubation contained a substantial number of fibrils after 2 weeks (Fig. 2c, 3-week aliquot), whereas far fewer fibrils were observed in the WT/A53T mixture (not shown). No fibrillar material was detected in the WT (Fig. 2a) or A30P (Fig. 2b) incubations after 3 weeks. However, the A30P incubation contained many spherical assemblies about $4 \mathrm{~nm}$ in height, which were possibly prefibrillar intermediates analogous to the $A \beta$ protofibril ${ }^{13}$. After incubation for 9 more weeks, both the WT (ref. 14) and A30P incubations contained fibrillar material (the comparative kinetics during this period were not foll owed). Even at a much lower concentration $(50 \mu \mathrm{M})$, A53T produced fibrillar material after 8 weeks of incubation (Fig. 2d). In addition to fib-
Fig. 1 Circular dichroism spectra. a, WT $\alpha$-synuclein and the two mutant proteins, A30P and $\mathrm{A} 53 \mathrm{~T}$, were mainly random coil in PBS. b. Helicity could be induced by the addition of $0.5 \%$ SDS or $10 \%$ HFIP (data not shown). The two mutant proteins, $\mathrm{A} 30 \mathrm{P}$ and $\mathrm{A} 53 \mathrm{~T}$, were indistinguishable from WT under these conditions.
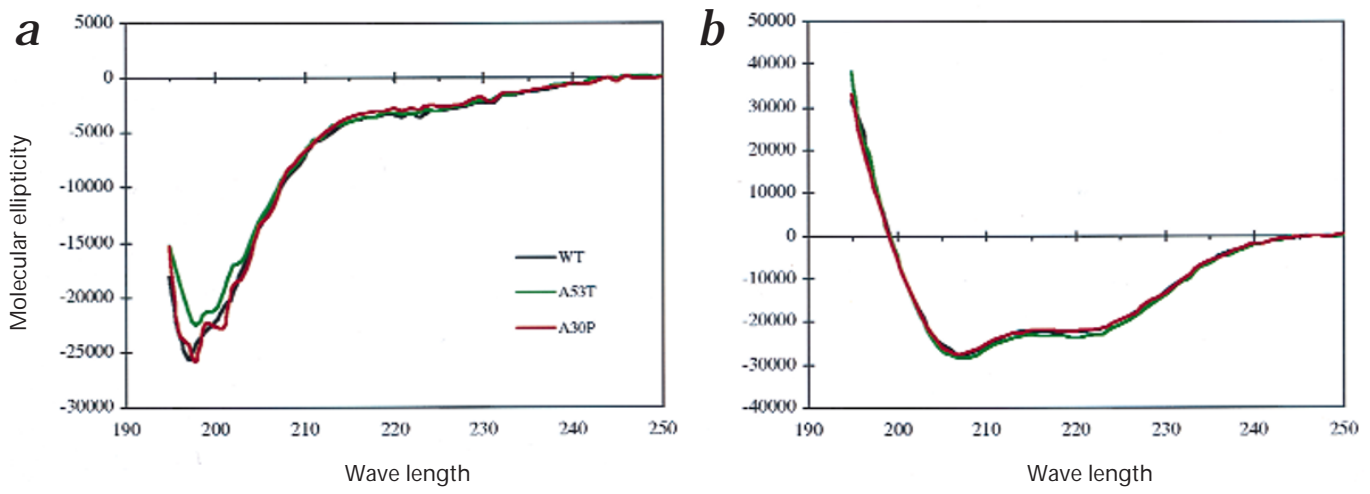
Fig. 2 Atomic force microscopy images. The mica surface appears dark brown, and the brightness of the synuclein-derived features increases as a function of their height; thus, the 8-10-nm peaks along the fibrils in c appear brighter than the 4-nm spheres. Solutions of $\alpha$-synuclein were incubated for 3 weeks at a concentration of $300 \mu \mathrm{M}$ (a, WT; $\mathbf{b}$, A30P; $\mathbf{c}$, A53T) or 8 weeks at 50 $\mu \mathrm{M}$ (d, A53T). Only small spherical species (heights usually 3-5 nm) were observed in WT (a) and A30P (b) solutions. In A53T solutions (c and d), fibrils 9-10 $\mathrm{nm}$ in height (red arrows) and filaments about $5 \mathrm{~nm}$ in height (green arrows) were commonly observed in addition to the small spherical species. The fibrils 9-10 nm in height had a left-handed helical twist with a periodicity (measured between adjacent increases in height) that was very regular within each individual fibril but which ranged between $40 \mathrm{~nm}$ and $175 \mathrm{~nm}$ among different fibrils (red arrows in $b$ indicate two periods of about $60 \mathrm{~nm}$ on one fibril). Scale bar represents $200 \mathrm{~nm}$ for all images. The shortness of the fibrils in c may be due to shearing of the gel during sample preparation.

rils, many spherical A53T assemblies $4 \mathrm{~nm}$ in height were observed, analogous to those seen with A30P, supporting the idea that these are intermediates in fibril formation.

To compare the fibrils formed in vitro (A53T, A30P and WT) with those in Lewy bodies, we analyzed them by atomic force microscopy and electron microscopy. Their dimensions and morphology were similar to those of Lewy bodies in brain slices of patients with PD (ref. 15), as measured by electron microscopy, and of $\alpha$-synuclein-containing fibrils extracted from cortical Lewy bodies characteristic of diffuse Lewy body disease ${ }^{16,17}$ and from filamentous inclusions characteristic of multiple system atrophy $^{18,19}$ (these all presumably contain only WT). The A53T, A30P and WT in vitro fibrils were $8-10 \mathrm{~nm}$ in height (by atomic force microscopy) and about $10 \mathrm{~nm}$ in width (by electron microscopy). Some of the A53T (Fig. 2c) and A30P fibrils (not shown) had a helical periodicity and were accompanied by filaments about $5 \mathrm{~nm}$ in height. Fibrils of PD Lewy bodies, observed in tissue slices, are reported to be about $10 \mathrm{~nm}$ in width ${ }^{15}$. Some fibrils extracted from brains of patients with diffuse Lewy body disease ${ }^{17}$ and multiple system atrophy ${ }^{18}$ are about $10 \mathrm{~nm}$ in width ('straight'; ref. 18) and others have a 'twisted' appearance (width varies between 5 and $18 \mathrm{~nm}$ ), two subfilaments ${ }^{17,18}$. Filaments of 5 $\mathrm{nm}$ width, in isolation and projecting from fibril termini, are also observed $^{17}$. The A53T fibrils formed in vitro also had a two-filament twisted morphology, by electron microscopy (Fig. 3a). The similarities in morphology indicate that fibrils formed in vitro reflect at least a subpopulation of fibrils formed during Lewy body disease. The twisted two-filament architecture of both the ex vivo and the in vitro fibrils containing $\alpha$-synuclein is reminiscent of $A \beta$ fibrils formed in vitro ${ }^{12}$, indicating that $\alpha$-synuclein fibril formation may also be a seeded polymerization ${ }^{6,10}$. The importance of distinct polymorphic fibrillar forms generated in vitro (J.H. and
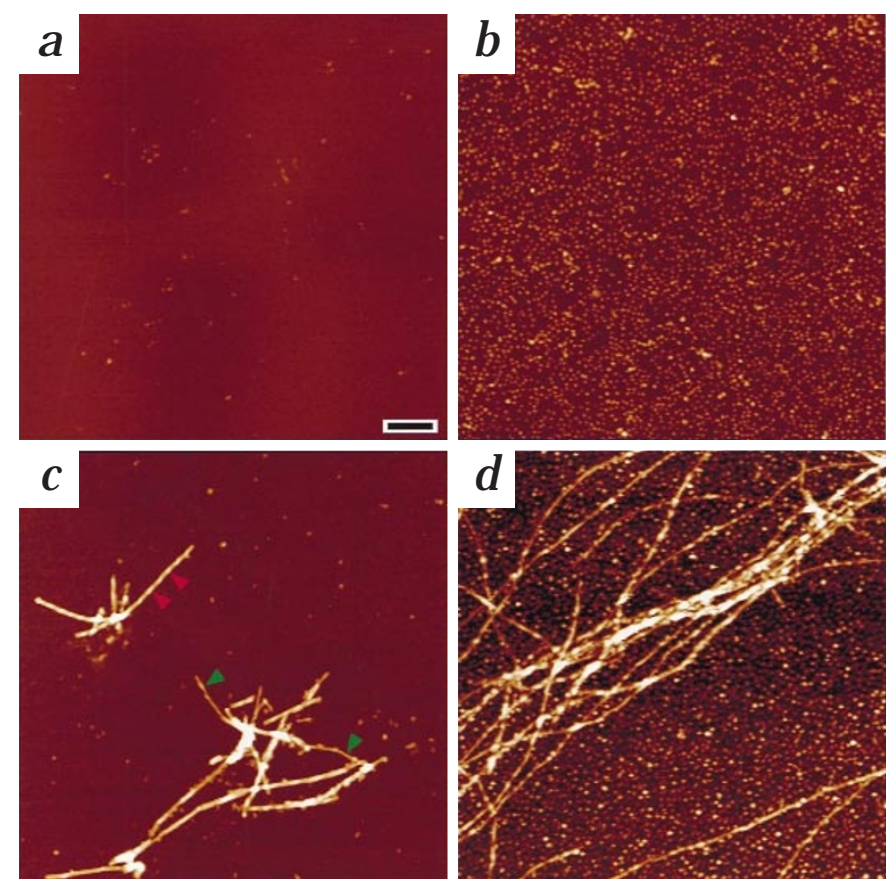

K.C., unpublished results) has not been assessed because the scarcity of brain tissue/Lewy bodies from familial PD cases has prevented direct comparison with ex vivo fibrils.

To further compare the A53T fibrils produced in vitro with those extracted from brains of patients with diffuse Lewy body disease and multiple system atrophy, we used immunogold electron microscopy, with three antibodies that have been used to stain Lewy bodies in PD ${ }^{4,20}$ and diffuse Lewy body disease brain tissue $^{17,20}$, and cytoplasmic inclusions in multiple system atrophy brain tissue ${ }^{18,19}$. An antibody raised against residues 1-10 of synuclein ${ }^{20}$ (TI17) labeled the sides of fibrillar A53T assemblies, as well as nonfibrillar, spherical A53T (Fig. 3b). The antibody PER4, which recognizes an indeterminant epitope in the $\alpha$-synuclein C-terminal domain ${ }^{17}$, similarly labeled A53T fibrils and spherical assemblies (not shown). In contrast, a monoclonal antibody $(\mathrm{H} 3 \mathrm{C})$ that recognizes an epitope at the extreme C-terminus ${ }^{21}$ stained spherical but not fibrillar A53T (Fig. 3c).

The data presented here indicate that the A53T mutation, and possibly the A30P mutation, may cause early-onset PD by accelerating $\alpha$-synuclein fibril formation, which may be an early step in Lewy body generation ${ }^{3,22}$. Acceleration of $\alpha$-synuclein fibril formation by another mechanism (for example, increased expression or decreased degradation) may explain the common idiopathic form
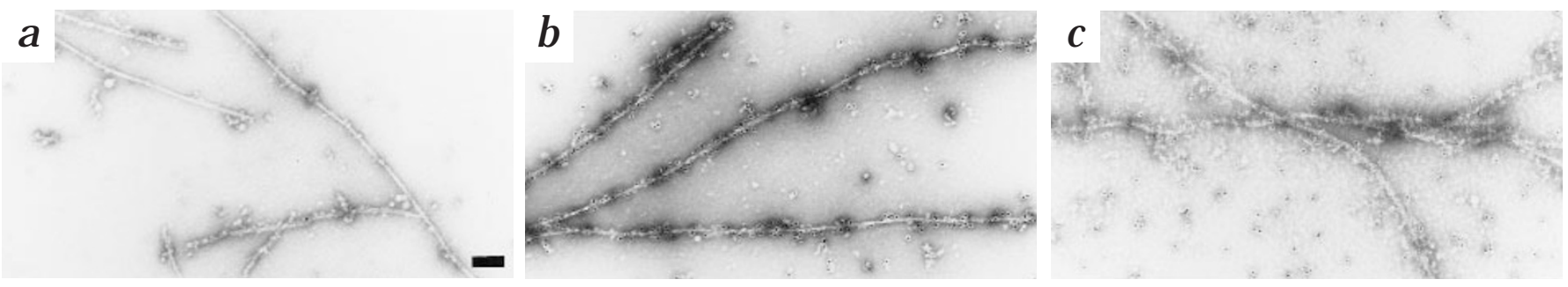

Fig. 3 Electron microscopy images. a, $\alpha$-Synuclein A53T fibrils, prepared from A53T incubated for 3 weeks at a concentration of $300 \mu \mathrm{M}$, were adsorbed to the electron microscopy membrane, stained with uranyl acetate and gold-conjugated secondary antibody. Original magnification, $\times 90,000$. b, A53T fibrils grown for 8 weeks at $50 \mu \mathrm{M}$. Immunogold electron microscopy with polyclonal antibody TI17 to the
$\mathrm{N}$-terminal epitope stains both fibrillar and nonfibrillar (globular) forms of $\alpha$-synuclein Original magnification, $\times 72,500$. c, A53T fibrils grown for 8 weeks at $50 \mu \mathrm{M}$. Immunogold electron microscopy with monoclonal antibody H3C to the C-terminal epitope stains only nonfibrillar $\alpha$-synuclein. Original magnification, $\times 72,500$. Scale bar represents $100 \mathrm{~nm}$ in a, and $125 \mathrm{~nm}$ in $\mathrm{b}$ and $\mathrm{c}$. 
of PD which is characterized by Lewy bodies containing wild-type $\alpha$-synuclein. Regardless of the underlying cause, inhibiting $\alpha$ synuclein fibrillization may be an effective therapeutic strategy against the disease. The in vitro system described here will allow compound libraries to be screened to identify $\alpha$-synuclein fibrillization inhibitors. Such compounds may allow the determination of whether it is the fibril itself or a precursor that is pathogenic. Our results also indicate that transgenic animals that overexpress the A53T mutant may be useful models of Parkinson disease.

\section{Methods}

$\alpha$-Synuclein mutagenesis. A pRK172 plasmid containing wild-type human $\alpha$-synuclein (pRK172/ $\alpha$ synWT, provided by R. Jakes and M. Goedert) was mutated using the QuickChange site-directed mutagenesis protocol (Stratagene, La Jolla, California). To create plasmid pRK172/ osynA53T, a Gto-A mutation was introduced into the plasmid at nucleotide 157, corresponding to an alanine-to-threonine mutation at position 53 of the protein sequence. To create plasmid pRK172/ asynA30P, a G-to-C mutation was introduced into the plasmid at nucleotide 88 , corresponding to an alanine to proline mutation at position 30 of the protein sequence. Mutagenesis was verified by $A B I 377$ Fluorescent DNA sequencing.

$\alpha$-Synuclein expression and purification. The pRK172/ $\alpha$ synWT plasmid was transfected into BL21(DE3) Eschericia coli and expression was induced by the addition of isopropyl $\beta$-D-thiogalactopyranoside. Cell lysate was precipitated in ammonium sulfate $\left(50 \%\right.$ saturation at $\left.0{ }^{\circ} \mathrm{C}\right)$. The pellet was resuspended, loaded onto a ProteinPak DEAE 5PW column $(21 \times 150 \mathrm{~mm}$; Waters, Milford, Massachusetts) in $10 \mathrm{mM}$ Tris, $\mathrm{pH} 7.4$ and eluted in a gradient of 0-700 mM $\mathrm{NaCl}$ over 70 minutes ( $\alpha$-synuclein eluted at about $300 \mathrm{mM} \mathrm{NaCl}$ ). Fractions containing $\alpha$-synuclein (analyzed by Coomassie-stained SDS-PAGE) were concentrated in an Ultrafree-15, 5K MWCO filter (Millipore, Bedford, Massachusetts), loaded onto a Sephadex G75 column $(10 \times 300 \mathrm{~mm}$; Pharmacia) and eluted in $100 \mathrm{mM} \mathrm{NH}{ }_{4} \mathrm{HCO}_{3}$. Fractions containing $\alpha$-synuclein were combined and lyophilized. All three proteins were determined to be about $95 \%$ pure by SDS-PAGE and electrospray mass spectrometry.

Circular dichroism spectroscopy. Samples were prepared by dissolving protein into PBS ( $10 \mathrm{mM} \mathrm{Na} \mathrm{HPO}_{4}, 2.7 \mathrm{mM} \mathrm{KCl}, 137 \mathrm{mM} \mathrm{NaCl}, \mathrm{pH} 7.4,25$ ${ }^{\circ} \mathrm{C}$ ); protein concentrations were determined by quantitative amino-acid analysis. Spectra were collected on an AVIV 62A DS spectrophotometer using a $0.1-\mathrm{cm}$ cell at room temperature.

In vitro fibrillization. Samples were dissolved in $10 \mathrm{mM} \mathrm{Na}_{2} \mathrm{HPO}_{4}, 100 \mathrm{mM}$ $\mathrm{NaCl}, \mathrm{pH} 7.4$; protein concentrations were determined by quantitative amino-acid analysis. Samples were incubated at $37^{\circ} \mathrm{C}$ without agitation.

Atomic force microscopy. This measures the position of a sensitive cantilever with a silicon probe tip as a sample adsorbed onto a smooth substrate (for example, mica) is 'rastered' underneath. The vertical movements required to maintain constant tip position (and applied force) are recorded throughout the scan, providing a digitized three-dimensional topograph of the specimen. Here, the digital data was converted into a two-dimensional image by assigning each point in the array a color representing the relative vertical movement of the specimen at that point.

Solutions of $\alpha$-synuclein were incubated without agitation at $37{ }^{\circ} \mathrm{C}$ in 10 $\mathrm{mM} \mathrm{Na}_{2} \mathrm{HPO}_{4}, 100 \mathrm{mM} \mathrm{NaCl}$ ( $\mathrm{pH}$ 7.4) for 3 weeks at a concentration of 300 $\mu \mathrm{M}$ or 8 weeks at $50 \mu \mathrm{M}$. The aggregation mixtures were mixed by gentle tapping and samples were prepared for imaging by placing $2 \mu \mathrm{L}$ of the incubation on top of $4 \mu \mathrm{L}$ of $250 \mathrm{mM} \mathrm{NaCl}, 250 \mathrm{mM} \mathrm{KCl}$ on the surface of freshly cleaved mica. After being incubated for $15 \mathrm{~min}$ under high-humidity conditions to prevent drying, the substrate was rinsed twice with $50 \mu \mathrm{L}$ water to remove salt and loosely bound peptide. Excess water was removed with a gentle stream of filtered compressed air, and the sample was imaged immediately. All images were obtained in ambient conditions using a Nanoscope Illa force microscope (Digital Instruments, Santa Barbara, California) operating in TappingMode ${ }^{\mathrm{TM}}$ with an etched silicon Nano Probe $^{\mathrm{TM}}$ (model FESP: $225 \mu \mathrm{m}$ cantilever; spring constant $=1-5 \mathrm{~N} / \mathrm{m}$; tip radius = 5-10 nm; Digital Instruments, Santa Barbara, California). Scanning parameters varied with individual tips and samples, but typical ranges were: starting RMS amplitude, 1.4-2.0 V; setpoint, 1.0-1.6 V; resonant frequency, $60-80 \mathrm{kHz}$; scan rate, $0.8-2 \mathrm{~Hz}$.

Electron microscopy. An aliquot from an incubation (10 $\mu \mathrm{l})$ was diluted 10fold, adsorbed onto Formvar-coated, carbon-stabilized copper grids (200 mesh) and air-dried. The grids were then rinsed briefly with $50 \mathrm{mM}$ Tris-HCl, $150 \mathrm{mM} \mathrm{NaCl}, \mathrm{pH} 7.4$ (TBS) and blocked with $1 \%$ egg albumin in the same buffer to reduce nonspecific background. Grids were then incubated with the primary antibody (monoclonal antibody H3C (from J. George and D. Clayton), polyclonal antibody PER4 (from M. Goedert), or polyclonal antibody TI17 (from T. Iwatsubo) at various dilutions from 1:400 to 1:2000), washed in TBS, and incubated again in colloid gold-conjugated goat antimouse IgG and goat anti-rabbit IgG secondary antibody (Amersham). Control grids were incubated with only secondary antibody. After brief fixation with $2.5 \%$ glutaraldehyde and rinsing with buffer, the grids were negatively stained with $2 \%$ aqueous uranyl acetate, air dried, and examined with a JEOL 100 CXII transmission electron microscope at an accelerating voltage of $80 \mathrm{kV}$.

\section{Acknowledgments}

We thank Y. Xu for doing the immunogold electron microscopy studies and C. Costello and R. Theberg for mass spectrometry. K.C. was supported by a National Science Foundation graduate fellowship.

\section{RECEIVED 13 JULY; ACCEPTED 29 SEPTEMBER 1998}

1. Kruger, R et al. Ala30Pro mutation in the gene encoding $\alpha$-synuclein in Parkinson's disease. Nature Genet. 18, 106-108 (1998).

2. Polymeropoulos, M.H. et al. Mutation in the $\alpha$-synuclein gene identified in families with Parkinson's disease. Science 276, 2045-2047 (1997).

3. Goedert, M. Familial Parkinson's disease. The awakening of alpha-synuclein [news]. Nature 388, 232-233 (1997).

4. Spillantini, M.G. et al. $\alpha$-synuclein in Lewy bodies. Nature 388, 839-840 (1997).

5. Weinreb, P.H., Zhen, W., Poon, A.W., Conway, K.A. \& Lansbury, P.T. NACP, a protein implicated in Alzheimer's disease and learning, is natively unfolded. Biochemistry $\mathbf{3 5}$, 13709-13715 (1996).

6. Harper, J.D. \& Lansbury, P.T. Models of amyloid seeding in Alzheimer's disease and scrapie: mechanistic truths and physiological consequences of the time-dependent solubility of amyloid proteins. Ann. Rev. Biochem. 66, 385-407 (1997).

7. Jarrett, J.T. \& Lansbury, P.T. Seeding "one-dimensional crystallization" of amyloid: a pathogenic mechanism in Alzheimer's disease and scrapie? Cell 73, 1055-1058 (1993).

8. Jarrett, J.T., Berger, E.P. \& Lansbury, P.T. The carboxy terminus of the beta amyloid protein is critical for the seeding of amyloid formation: implications for the pathogenesis of Alzheimer's disease. Biochemistry 32, 4693-4697 (1993).

9. Selkoe, D. Alzheimer's disease: genotypes, phenotype, and treatments. Science $\mathbf{2 7 5}$, 630-631 (1997).

10. Lansbury, P.T. Structural Neurology: Are seeds at the root of neuronal degeneration? Neuron 19, 1151-1154 (1997).

11. Davidson, W.S., Jonas, A., Clayton, D.F. \& George, J.M. Stabilization of $\alpha$-synuclein secondary structure upon binding to synthetic membranes. J. Biol. Chem. 273, 9443-9449 (1998).

12. Harper, J.D., Lieber, C.M. \& Lansbury, P.T. Atomic force microscopic imaging of seeded fibril formation and fibril branching by the Alzheimer's disease amyloid-- protein. Chem. Biol. 4, 951-959 (1997).

13. Harper, J.D., Wong, S.S., Lieber, C.M. \& Lansbury, P.T. Observation of metastable A $\beta$ amyloid protofibrils by atomic force microscopy. Chem. Biol. 4, 119-125 (1997).

14. Hashimoto, M. et al. Human recombinant NACP/alpha-synuclein is aggregated and fibrillated in vitro: relevance for Lewy body disease. Brain Res 799, 301-306 (1998).

15. Forno, L.S. \& Langston, J.W. Lewy Bodies and Aging: Relation to Alzheimer's and Parkinson's Diseases. N eurodegeneration. 2, 19-24 (1993).

16. Kosaka, K. \& Iseki, E. Dementia with Lewy bodies. Curr. Opin. Neurol. 9, 271-275 (1996).

17. Spillantini, M.G., Crowther, R., Jakes, R., Hasegawa, M. \& Goedert, M. $\alpha$-Synuclein in filamentous inclusions of Lewy bodies from Parkinson's disease and dementia with Lewy bodies. Proc. Natl. Acad. Sci. USA 95, 6469-6473 (1998).

18. Spillantini, M.G. et al. Filamentous alpha-synuclein inclusions link multiple system atrophy with Parkinson's disease and dementia with Lewy bodies. Neurosci. Lett. 251, 205-208 (1998).

19. Tu, P.-h. et al. Glial cytoplasmic inclusions in white matter oligodendrocytes of multiple system atrophy brains contain insoluble $\alpha$-synuclein. Ann. N eurol. 44, 415-422 (1998).

20. Baba, M. et al. Aggregation of $\alpha$-synuclein in Lewy bodies of sporadic Parkinson's dis ease and dementia with Lewy bodies. Am. J. Pathol. 152, 879-884 (1998).

21. George, J.M., Jin, H., Woods, W.S. \& Clayton, D.F. Characterization of a novel protein regulated during the critical period for song learning in the zebra finch. Neuron 15, 361-372 (1995).

22. Trojanowski, J. \& Lee, V. Aggregation of neurofilament and alpha-synuclein proteins in Lewy bodies: implications for the pathogenesis of Parkinson disease and Lewy body dementia. Arch. Neurol. 55, 151-152 (1998). 